\title{
Modeling IR spectra of OH/IR stars at different phases ${ }^{\star}$
}

\begin{abstract}
Kyung-Won Suh and H.-Y. Kim
Department of Astronomy and Space Science, Chungbuk National University, Cheongju-City, 361-763, Republic of Korea

Received 10 April 2002 / Accepted 6 June 2002

Abstract. We investigate the spectral energy distributions (SEDs) of OH/IR stars (OH127.8+0.0 and OH26.5+0.6) having thick dust envelopes at different pulsation phases. Using new infrared observational data including the Infrared Space Observatory (ISO) data, we determine the new pulsation parameters. The deep silicate absorption features show significant variations depending on the pulsation phase. The variations are mainly due to changes in the properties of dust envelopes around the $\mathrm{OH} / \mathrm{IR}$ stars. Comparing the results of detailed radiative model calculations with observations, we explore the changes of the relevant parameters of the envelopes and central stars depending on the pulsation phase. We find that when the central luminosity increases from the minimum to maximum phase, the inner radius of the dust shell increases with velocity faster than the outer shell expansion velocity and the dust shell optical depth decreases. During the phase change from the minimum to maximum, we find that dust formation ceases and about a half of the dust grains in the volume difference should have evaporated. During the phase change from the maximum to minimum, we find that the dust formation should be enhanced because the inner radius is decreasing. In the outer radii of the dust shell, the constant dust winds are easily maintained. We expect that the dust evaporation process driven by pulsation could be a mechanism for crystallizing the dust grains in inner regions of the dust shells around $\mathrm{OH} / \mathrm{IR}$ stars.
\end{abstract}

Key words. radiative transfer - stars: AGB and post-AGB - stars: circumstellar matter - stars: individual: $\mathrm{OH} 26.5+0.6$, $\mathrm{OH} 127.8+0.0$

\section{Introduction}

The OH/IR stars are generally considered to be the final phase of an oxygen-rich asymptotic giant branch (AGB) star before leaving the red giant branch and rapidly evolving into a planetary nebula. This phase of the Mira variable is characterized by the long period, large amplitude pulsation and dusty stellar winds with high mass-loss rates $\left(10^{-7}-10^{-4} M_{\odot} / \mathrm{yr}\right)$. Although the $\mathrm{OH} / \mathrm{IR}$ stars are pulsating, the outer region of the dust shells show constant expansion velocity $\left(v_{\text {exp }} \sim 10-30 \mathrm{~km} \mathrm{~s}^{-1}\right)$. This implies that almost constant mass loss rate is maintained for a time scale much larger than the pulsation periods (e.g. Herman \& Habing 1985).

For many pulsating AGB stars, it has been known that the shapes of SEDs vary as well as the overall luminosity depending on the phase of pulsation (e.g. Forrest et al. 1978; Le Bertre 1988). There should be important implications to be drawn from the variations of SEDs: the shapes are affected by the parameters of the dust shells as well as the central stars, depending on the phase of pulsation.

Send offprint requests to: Kyung-Won Suh,

e-mail: kwsuh@chungbuk.ac.kr

* Based on observations with ISO, an ESA project with instruments funded by ESA Member States (especially the PI countries: France, Germany, The Netherlands and the UK) and with the participation of ISAS and NASA.
Le Bertre (1988) modeled the dust shell of a carbon Mira R For as a function of pulsation. Le Sidaner \& Le Bertre (1993) modeled two oxygen-rich Miras with thin dust shells as a function of phase. Monnier et al. (1998) investigated temporal variations of mid-infrared spectra in a number of late-type stars including several oxygen-rich AGB stars. Lobel et al. (2000) modeled two oxygen-rich Miras with thin dust shells. All the above authors investigated the SEDs of the low mass-loss rate O-rich AGB stars with emission at 10 and $18 \mu \mathrm{m}$.

Suh et al. (1990) modeled OH26.5+0.6 with absorption at 10 and $18 \mu \mathrm{m}$ depending on the phase using the results of a pulsation shock model. The photometric study by Jones et al. (1990) investigated the pulsation properties of many OH/IR stars. Now, new observational data including ISO have provided valuable opportunity to improve our knowledge of the pulsating $\mathrm{OH} / \mathrm{IR}$ stars with thick dust shells.

In this paper, we investigate the SEDs of two OH/IR stars $(\mathrm{OH} 127.8+0.0$ and $\mathrm{OH} 26.5+0.6)$ with thick dust envelopes as a function of pulsation phase. We obtain detailed parameters of the dust shell as well as the central star depending on the pulsation phase, paying close attention to the observed SEDs at different phases. The dust shells around $\mathrm{OH} / \mathrm{IR}$ stars can be a good laboratory to understand formation and destruction processes of dust grains in a cycle of thermal pulse. The purpose of this paper is to investigate the physics and chemistry of dust grains and dust shells as a function of pulsation phase and find 
Table 1. Observational data for light curves.

\begin{tabular}{llllll}
\hline \hline $\begin{array}{l}\text { Name } \\
\text { (IRAS PSC) }\end{array}$ & $\begin{array}{l}K \text { band } \\
\lambda \text { (number; unused) }\end{array}$ & $\begin{array}{l}L \text { band } \\
\lambda \text { (number) }\end{array}$ & $\begin{array}{l}M \text { band } \\
\lambda \text { (number; unused) }\end{array}$ & $\begin{array}{l}\text { Obs. date } \\
(J D 2440 \text { 000+) }\end{array}$ & Reference. \\
\hline OH127.8+0.0 & $2.3(3)$ & $3.6(3)$ & $4.9(3)$ & $2696-4612$ & Gehrz et al. (1985) \\
$(01304+6211)$ & - & $3.58(1)$ & $4.9(1)$ & 3040 & Ney \& Merrill (1980) \\
& $2.3(2)$ & $3.6(2)$ & $4.9(2)$ & 4437,4604 & Grasdalen et al. (1983) \\
& $2.2(2)$ & $3.6(2)$ & $4.9(2)$ & 5601,7493 & Persi et al. (1990) \\
& $2.2(2)$ & $3.5(10)$ & $4.8(10)$ & $7052-7503$ & Jones et al. (1990) \\
& - & $3.5(1)$ & $4.8(1)$ & 10825 & ISO SWS01 \\
OH26.5+0.6 & - & $3.5(1)$ & $4.8(1)$ & 10471 & ISO PHT40 \\
$(18348-0526)$ & $2.28(10 ; 1)$ & $3.5(10)$ & $4.9(4)$ & $2171-3255$ & Ney \& Merrill (1980) \\
& $2.2(2)$ & $3.4(3)$ & $4.8(3)$ & $2230-2565$ & Evans II \& Beckwith (1977) \\
& - & $3.5(1)$ & $4.9(1)$ & 2270 & Lebofsky et al. (1978) \\
& $2.2(11 ; 1)$ & $3.7(11)$ & $4.8(10)$ & $2817-4533$ & Engels (1982) \\
& - & $3.4(2)$ & $4.8(1)$ & 2905,2557 & Forrest et al. (1978) \\
& $2.2(1)$ & $3.4(1)$ & $4.8(1)$ & 3691 & Werner et al. (1980) \\
& $2.3(1)$ & $3.6(1)$ & $4.9(1)$ & 4436 & Grasdalen et al. (1983) \\
& $2.2(1)$ & $3.5(2)$ & $4.8(2)$ & 4696 & Willems \& de Jong (1982) \\
& $2.2(2)$ & $3.5(11)$ & $4.8(11 ; 1)$ & $6164-7405$ & Jones et al. (1990) \\
& $2.19(1)$ & $3.79(1)$ & $4.64(1)$ & 8042 & Nyman et al. (1993) \\
& $2.2(1)$ & - & - & 8348 & Xiong et al. (1994) \\
$2.19(1)$ & $3.78(1)$ & $4.66(1)$ & 8378 & Lepine et al. (1995) \\
& $2.2(8)$ & $3.45(8)$ & - & $8420-9492$ & Olivier et al. (2001) \\
& - & $3.5(1)$ & $4.8(1)$ & 10368 & ISO SWS01 \\
\hline
\end{tabular}

the meanings. We present detailed values of global dust shell parameters at different phases using simple approximations and discuss the implications of the results.

\section{The light curves for the program stars}

For this paper, we choose two OH/IR stars having thick dust envelopes: $\mathrm{OH} 127.8+0.0$ and $\mathrm{OH} 26.5+0.6$. New observational data have become available since many authors investigated the pulsation properties of the $\mathrm{OH} / \mathrm{IR}$ stars. To complete the light curves in the bandpasses of $K(2.2 \mu \mathrm{m}), L(3.5 \mu \mathrm{m})$ and $M$ $(4.8 \mu \mathrm{m})$, we collect the data from the Fifth Catalog of Infrared Observations (Gezari et al. 2000) and the references therein and the new ISO data.

Table 1 lists the data used for this paper. Although the exact wavelengths for each bandpass used by many authors are slightly different, we use the original data without further reduction. There should be minor uncertainties for the differences. For curve-fitting of the observational data, we have used the Marquardt-Levenberg algorithm to find the coefficients of the independent variables that give the best fit between the sinusoidal equation and the data (e.g. Press et al. 1986). By analyzing the light curves, we determine new pulsation parameters: the period and amplitude. When data points are too far from the general trend, they are not used for determination of the pulsation parameters. Table 1 lists the number of available observational data and the number of unused data when applicable.

Figures 1 and 2 show the light curves for the two stars observed for about last 25 years covering various numbers of the pulsation phase cycle. In Fig 2, the three data points unused for the light curve fitting are also marked. We find that the usable
ISO data are nearly at the minimum phase for both of the objects. The light curves in $L$ bandpass produce minimal overall errors for the pulsation periods and amplitudes. Therefore, we adopt the values determined from the $L$ bandpass light curve as the parameters for further investigation. Table 2 lists the new pulsation parameters obtained in this paper and the parameters determined by previous authors.

\section{The model SED calculations}

We use the radiative transfer code developed by Egan et al. (1988) for spherically symmetric dust shells around OH/IR stars. Silicates are believed to be a major component of the dust grains in the envelopes around O-rich AGB stars. A fixed model parameter for the dust envelope is the wavelength dependence of the dust opacity. The adjustable input parameters are the dust optical depth at $10 \mu \mathrm{m}\left(\tau_{10}\right)$. We have performed the model calculations in the wavelength range 0.01 to $36000 \mu \mathrm{m}$.

\subsection{Central stars}

For the central star, we use the luminosity $\left(L_{*}\right)$ at minimum phase of $1 \times 10^{4} L_{\odot}$. A change in the luminosity does not affect the shape of the output SED, it only affects the overall energy output.

In this paper, we use the stellar black body temperature $\left(T_{*}\right)$ of $2000 \mathrm{~K}$ for all the models. Although the stellar surface temperature varies depending on the pulsation phase, we fix the temperature because the variations produce only minor deviations for all the models used in this paper. For the models with optically thin dust shells, a change in the blackbody 
Kyung-Won Suh and H.-Y. Kim: IR spectra of OH/IR stars at different phases
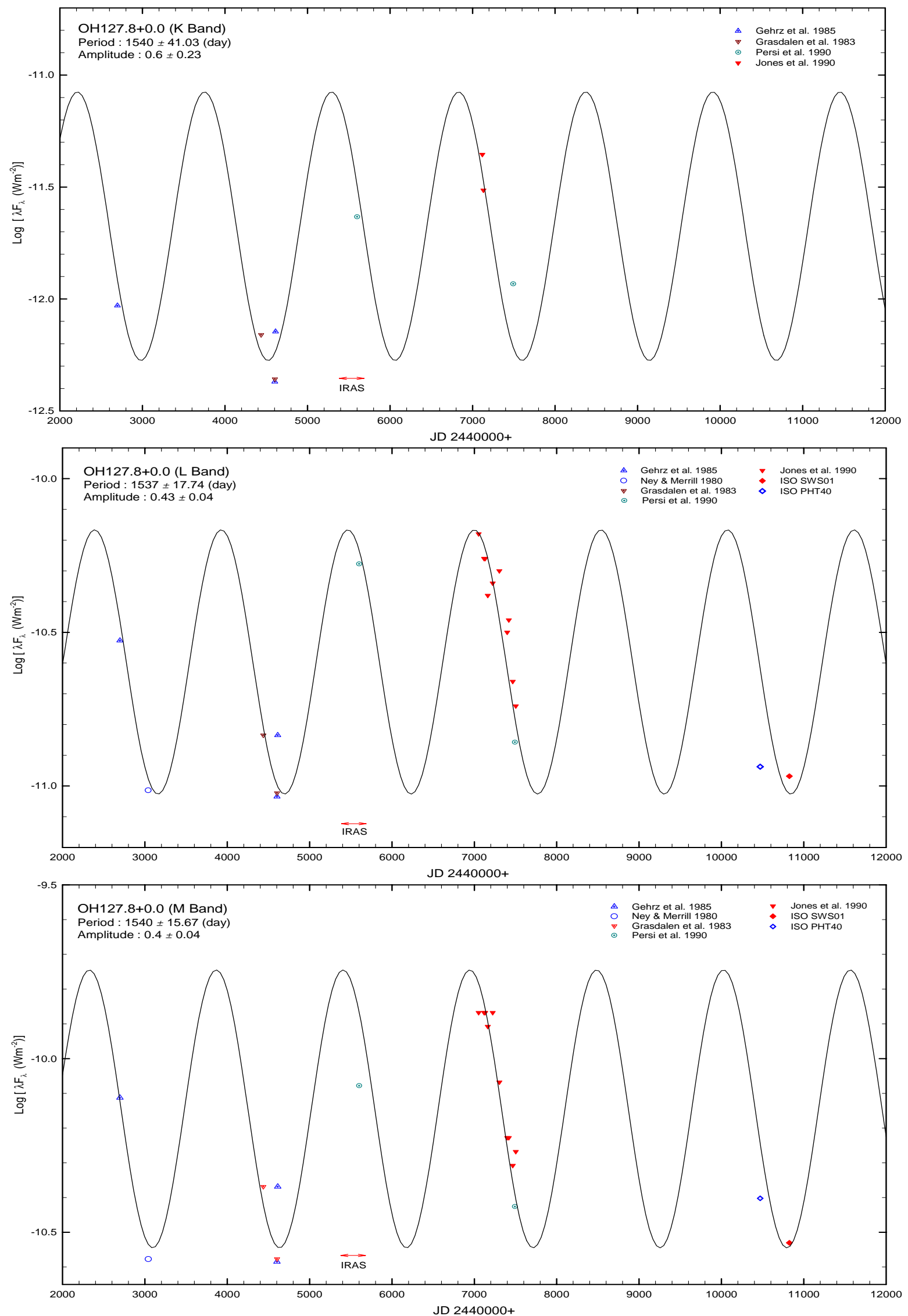

Fig. 1. Light curves for $\mathrm{OH} 127.8+0.0$. 

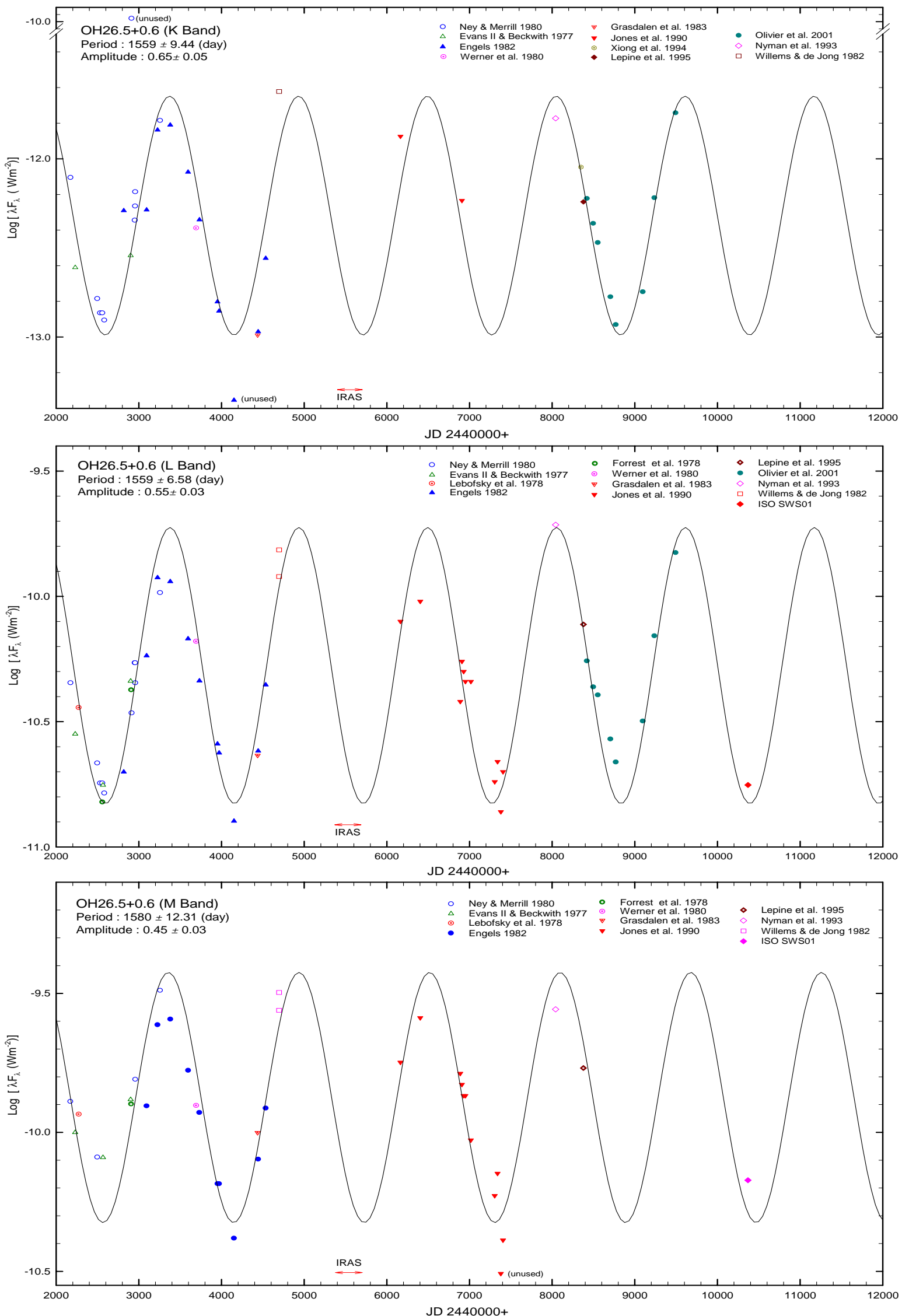

Fig. 2. Light curves for $\mathrm{OH} 26.5+0.6$. 
Table 2. Pulsation properties.

\begin{tabular}{llll}
\hline \hline Name & Period (days; Ref.) & $\begin{array}{l}\text { Amplitude at } L \text { band } \\
2.5 \log \left(\frac{F_{\max }}{F_{\min }}\right)\end{array}$ & $v_{\exp }\left(\mathrm{km} \mathrm{s}^{-1}\right)$ \\
\hline OH127.8+0.0 & $1994 \pm 130$ (Herman \& Habing 1985) & & 11 (Herman \& Habing 1985) \\
& & & 14 (Groenewegen et al. 1999) \\
& $1537 \pm 17.7$ (This paper) & $2.15 \pm 0.20$ (This paper) & 12.5 (Adopted for this paper) \\
OH26.5+0.6 & $1556 \pm 16$ (Herman \& Habing 1985) & 2.43 (Engels 1982) & 14 (Dyck et al. 1984) \\
& 1575 (Jones et al. 1990) & 2.5 (Jones et al. 1990) & 13.9 (Nyman et al. 1993) \\
& $1559 \pm 6.6$ (This paper) & $2.75 \pm 0.15$ (This paper) & 14 (Adopted for this paper) \\
\hline
\end{tabular}

temperature of the central star does affect the output spectra. However, for the models with optically thick dust shells, the changes of the black body temperature in the range $T_{*}=1800$ $2200 \mathrm{~K}$ produce only minor deviations in output SEDs. By fixing the uncertain stellar temperature, we may have a better chance to determine the variations of dust shell parameters.

\subsection{Dust shells}

We assume that the dust condensation temperature $\left(T_{\mathrm{c}}\right)$ is $1000 \mathrm{~K}$. We assume that the dust shell density distribution is continuous $\left(\rho \propto r^{-2}\right)$ from the dust condensation radius ( $R_{\mathrm{c}}$; inner radius of the dust shell) to the outer radius which is $10000 R_{\mathrm{c}}$. See Suh (1999) for the detailed discussion on these parameters.

Suh (1999) determined two opacity functions of amorphous silicates which fit AGB stars best: the warm dust opacity function for the stars with the $10 \mu \mathrm{m}$ emission features and the cool dust opacity function for the more dusty AGB stars with the $10 \mu \mathrm{m}$ absorption features. We use dust opacity function of amorphous cool silicates obtained by Suh (1999) and the opacity functions of crystalline silicates obtained by Jäger et al. (1998). We assume that the relative contents of crystalline enstatite and forsterite are the same.

For the dust grain opacity of a mixture of amorphous and crystalline silicates, we use the averaged single grain population model that treats the mixture as single-type grains whose properties average the actual mix.

Suh (2002) modeled eight oxygen-rich AGB stars using amorphous and crystalline silicates. The crystallinity $\alpha$, which is the relative content of crystalline silicates to all silicates, is defined as

$\alpha=\frac{\text { total mass of crystalline silicates }}{\text { total mass of amorphous and crystalline silicates }}$.

For simplicity, we assume that all silicate dust grains are spherical with a uniform radius of $0.1 \mu \mathrm{m}$ and the bulk density is $3.0 \mathrm{~g} \mathrm{~cm}^{-3}$. Depending on $\alpha$, we can easily calculate the opacity functions of the mixed silicates by simply manipulating the absorption efficiency functions of amorphous and crystalline silicates. Various values of the crystallinity $(\alpha=0,0.1,0.2$, 0.4 ) are used for model calculations. The crystallinity $\alpha=0$ means pure amorphous silicates.

\section{SED comparison}

To compare our model results with observed SEDs, we use the data from the IRAS PSC (4-color photometric data; $\lambda=12,25$, $60,100 \mu \mathrm{m}$ ), IRAS LRS (Low resolution spectral data $\lambda=8$ $22 \mu \mathrm{m})$ and other ground based and airborne observations. We also use ISO SWS $(\lambda=2.4-45.2 \mu \mathrm{m})$ and ISO LWS $(\lambda=45-$ $196.8 \mu \mathrm{m})$ data.

Unfortunately, for both program stars, we are not able to construct meaningful multiple phase spectra from ISO data. Therefore, we use the single set of data with best quality as presented by Sylvester et al. (1999) among multiple sets of available ISO SWS and LWS data of each program star. For SED comparison, we have used the reduced ISO SWS and LWS data from Sylvester et al. (1999).

Figures 3 and 4 displays the SED comparison of observations and model results at three different phases for the two stars. The legends of the observational data within each figure represent the reference and the time of the observation when available.

Figure 3 shows the SEDs of OH127.8+0.0. This star shows tremendous changes of bolometric luminosity over a pulsation cycle. We present the best fit models at wide wavelength range. The fit is good at the minimum phase. At the intermediate and maximum phases, the models do not fit observations well, especially at around $8-9 \mu \mathrm{m}$. We think this is due to lack of good available data. The $20 \%$ mixture model SEDs show the prominent crystalline features at 19.5, 23.1, 27.3, 33.3, 40.6 and $43.3 \mu \mathrm{m}$. The ISO spectra at minimum phase show prominent crystalline emission features at $33.3,40.6$ and $43.3 \mu \mathrm{m}$. To reproduce the emission features, we need a high value of crystallinity $(\alpha=0.2)$.

Figure 4 shows the SEDs of OH26.5+0.6. The upper panel shows the comparison at three phases, where the models for the minimum and intermediate phases fit the observations by Forrest et al. (1978) fairly well. The lower panel shows the ISO data compared with the model for minimum phase. Again, the ISO spectra at minimum phase show prominent crystalline emission features at 33.3, 40.6 and $43.3 \mu \mathrm{m}$. To reproduce the emission features, we need a high value of crystallinity $(\alpha=0.2)$.

For both of the objects, it is clear that the dust optical depth becomes smaller as the luminosity of central star increases. We expect evaporation of a significant amount of dust grains in the inner portion of the dust shell when total luminosity increases. 


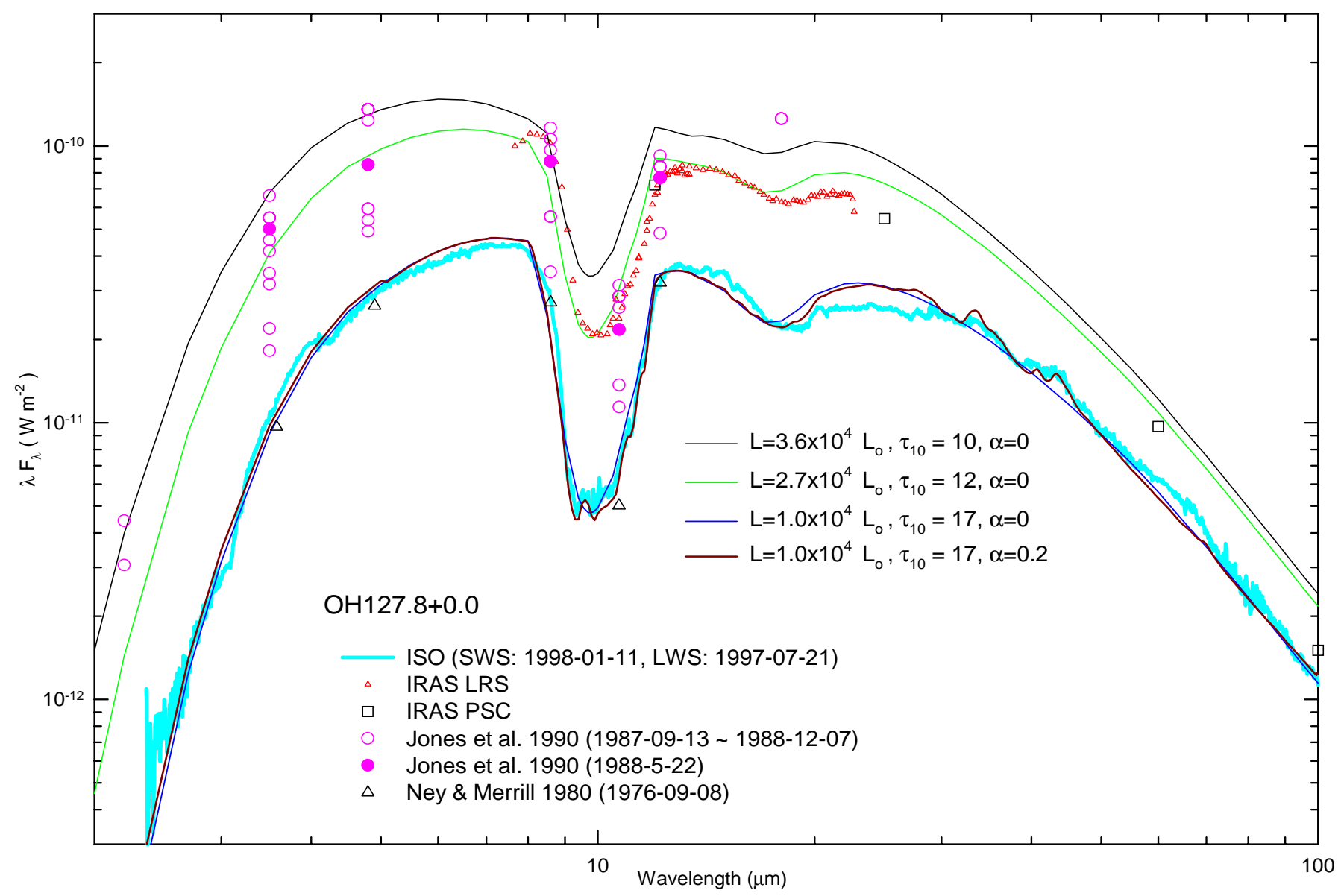

Fig. 3. SEDs at different phases for OH127.8+0.0.

Table 3 lists SED model fitting parameters. For each star, the table lists the pulsation phase, the optical depth, the central luminosity, the dust condensation radius $\left(R_{\mathrm{c}}\right)$, the dust number density at $R_{\mathrm{c}}$, the material density at $R_{\mathrm{c}}$, and the approximate mass-loss rate.

We assume that the mass-loss rate $(\dot{M})$ and the envelope expansion velocity $(v)$ are constant and density distribution is continuous $\left(\rho \propto r^{-2}\right)$ from the dust condensation radius $\left(R_{\mathrm{c}}\right)$. Under this assumption, the mass-loss rate is given by

$\dot{M}=4 \pi r^{2} \rho v \simeq 4 \pi R_{\mathrm{c}}^{2} \rho v$,

where $\rho$ is the material density at $R_{\mathrm{c}}$ that is given by

$\rho=\frac{4 \pi a^{3}}{3} \rho_{\mathrm{b}} n_{\mathrm{d}} \beta$

where $\rho_{\mathrm{b}}$ is the dust grain bulk density, $n_{\mathrm{d}}$ is the dust number density at $R_{\mathrm{c}}$ and $\beta$ is the gas to dust ratio which is given by

$\beta=\frac{\text { total material density }}{\text { total dust grain density }}$.

To estimate the material density and mass-loss rate, we assume that the bulk density $\left(\rho_{b}\right)$ of the silicate dust grains is $3.0 \mathrm{~g} \mathrm{~cm}^{-3}$, the gas to dust ratio $(\beta)$ is 100 , and the expansion velocity as listed in Table 2 . The calculated mass-loss rates are reasonably in the range of the observed values (e.g. Loup et al. 1993; Le Sidaner \& Le Bertre 1996; Sylvester et al. 1998).
For a given optical depth, the mass-loss rates with different values of the crystallinity $(\alpha)$ show only minor deviations.

Because constant expansion velocity $\left(v_{\text {exp }}\right)$ winds are found in $\mathrm{OH} / \mathrm{IR}$ stars, the mass-loss rate depends weakly on the pulsation phase. The deviations in Table 3 could be due to the uncertainty in model fitting with observations. We discuss about this point in next section.

\section{Physical changes of a dust shell depending on the pulsation phase}

\subsection{Basic parameters and the density distribution}

As an AGB star goes through a pulsation cycle, the physics and chemistry of the dust shell change as well as those of the central star. From Table 3, it is clear that dusts optical depth becomes smaller because dust shell inner radius gets larger as the luminosity of central star increases. Figure 5 shows the distribution of the dust density at the inner shell radius for different pulsation phases derived from model fitting with observations (Table 3 ). The overall shapes closely follow the continuous density distribution ( $r^{-2}$ density laws). Small deviations could be due to difficulties in fitting all the observations at different phases.

Radiative transfer models for the dust shells around AGB stars have been developed by a number of authors with various assumptions on input parameters and differing degrees of 

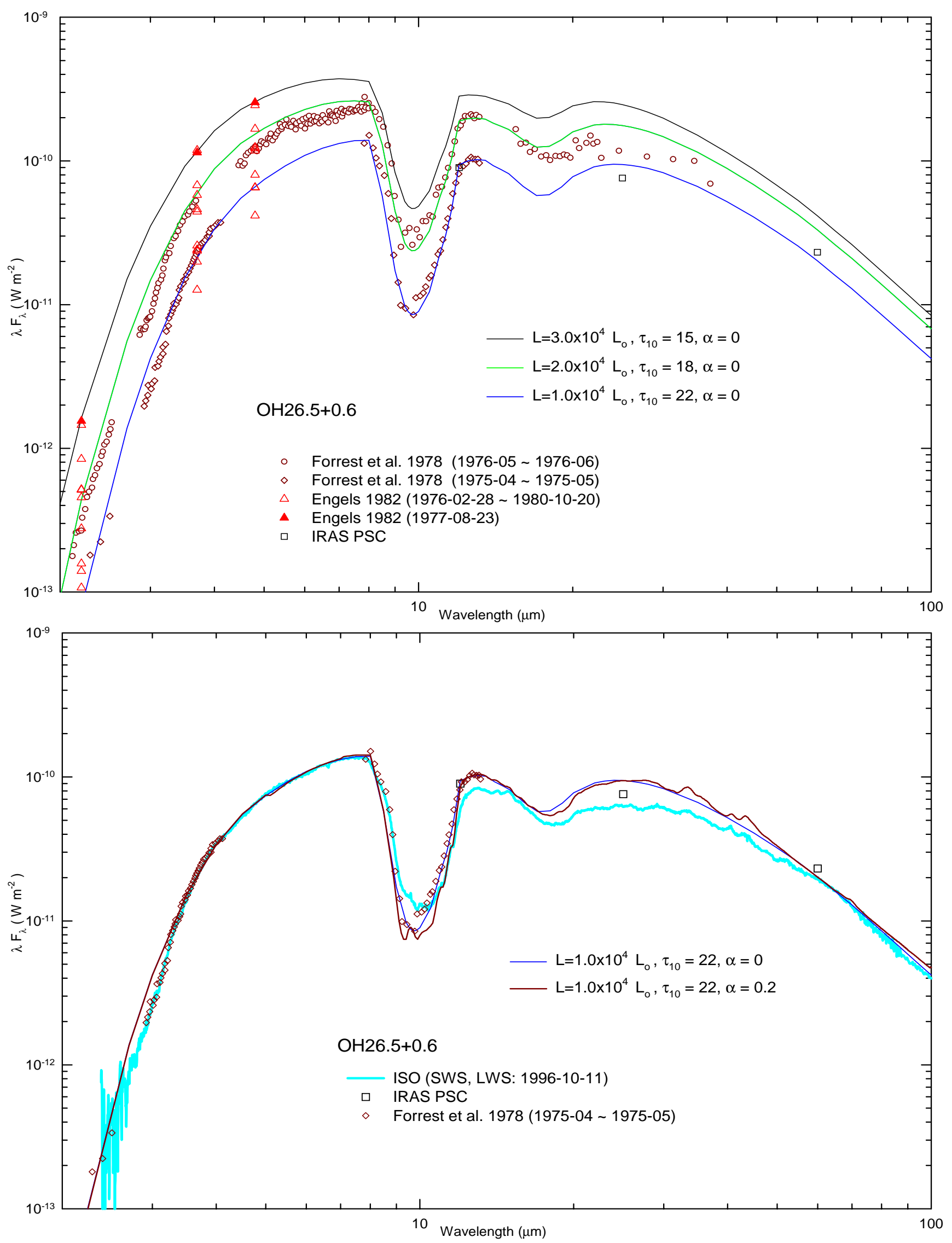

Fig. 4. SEDs at different phases for $\mathrm{OH} 26.5+0.6$. 
Table 3. Model SED fitting parameters and derived dust shell parameters. All models are for pure amorphous silicates $(\alpha=0)$.

\begin{tabular}{cccccccc}
\hline \hline $\begin{array}{c}\text { Object } \\
\text { Name }\end{array}$ & Phase & $\tau_{10 \mu \mathrm{m}}$ & $\begin{array}{c}L_{*} \\
\left(L_{\odot}\right)\end{array}$ & $\begin{array}{c}R_{\mathrm{c}} \\
(\mathrm{pc})\end{array}$ & $\begin{array}{c}n_{\mathrm{d}} \\
\left(\sharp \mathrm{cm}^{-3}\right)\end{array}$ & $\begin{array}{c}\rho \\
\left(\mathrm{g} \mathrm{cm}^{-3}\right)\end{array}$ & $\begin{array}{c}\dot{M} \\
\left(M_{\odot} / \mathrm{yr}\right)\end{array}$ \\
\hline OH127.8+0.0 & Minimum & 17 & $1.0 \mathrm{E}+04$ & $1.08 \mathrm{E}-04$ & $1.17 \mathrm{E}-03$ & $1.47 \mathrm{E}-15$ & $4.06 \mathrm{E}-05$ \\
& Intermediate & 12 & $2.7 \mathrm{E}+04$ & $1.71 \mathrm{E}-04$ & $5.27 \mathrm{E}-04$ & $6.58 \mathrm{E}-16$ & $4.53 \mathrm{E}-05$ \\
& Maximum & 10 & $3.6 \mathrm{E}+04$ & $1.93 \mathrm{E}-04$ & $3.85 \mathrm{E}-04$ & $4.84 \mathrm{E}-16$ & $4.27 \mathrm{E}-05$ \\
$\mathrm{OH} 26.5+0.6$ & Minimum & 22 & $1.0 \mathrm{E}+04$ & $1.12 \mathrm{E}-04$ & $1.46 \mathrm{E}-03$ & $1.84 \mathrm{E}-15$ & $6.11 \mathrm{E}-05$ \\
& Intermediate & 18 & $2.0 \mathrm{E}+04$ & $1.54 \mathrm{E}-04$ & $8.69 \mathrm{E}-04$ & $1.09 \mathrm{E}-15$ & $6.87 \mathrm{E}-05$ \\
& Maximum & 15 & $3.0 \mathrm{E}+04$ & $1.84 \mathrm{E}-04$ & $6.06 \mathrm{E}-04$ & $7.62 \mathrm{E}-16$ & $6.84 \mathrm{E}-05$ \\
\hline
\end{tabular}
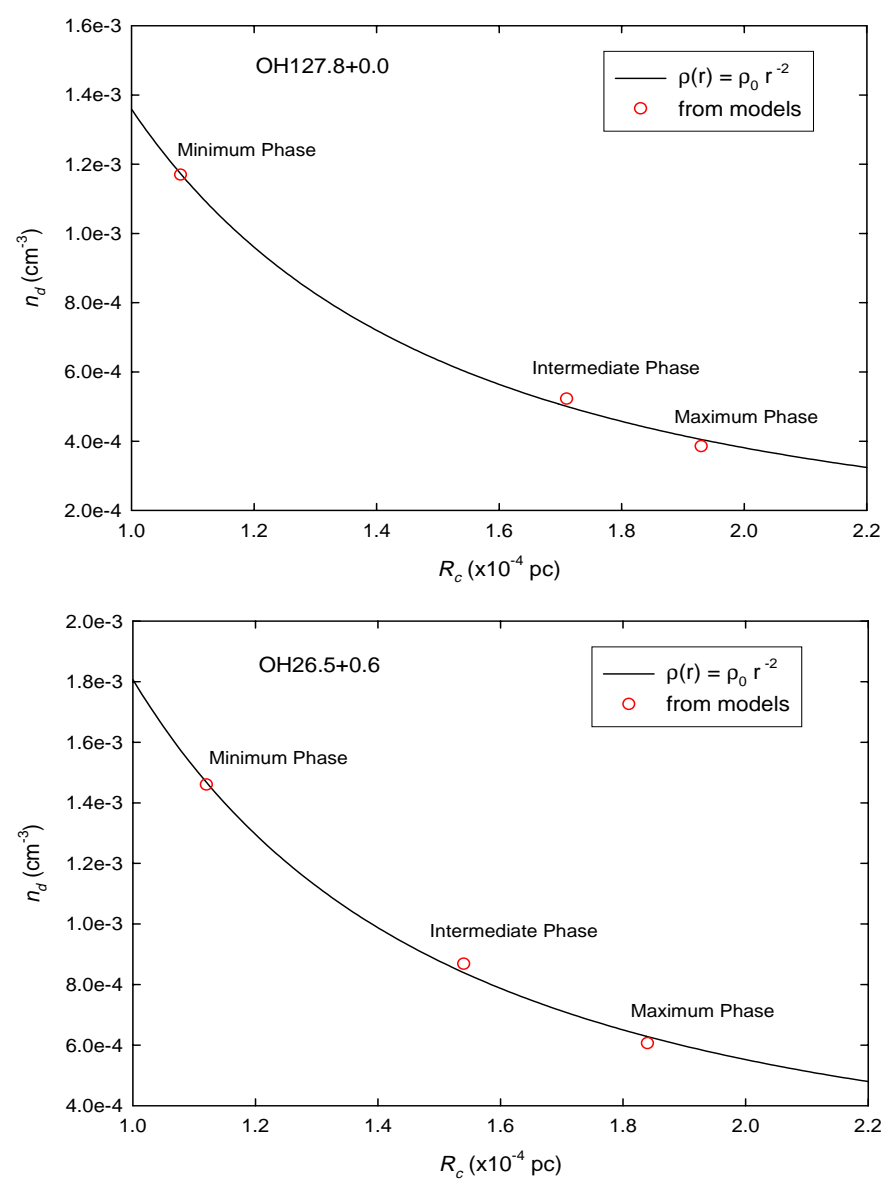

Fig. 5. Distribution of the dust number density at dust condensation radius for different phases.

sophistication (e.g. Jones \& Merrill 1976; Rowan-Robinson \& Harris 1983; Bedijn 1987; Volk \& Kwok 1988; Suh 1999). All these models assumed a smoothly distributed spherically symmetric dust shell with a power law description of the radial density distribution. The dust density distribution was most often taken to be inversely proportional to the square of the distance, as would be expected for the constant expansion velocity $\left(v_{\exp }\right)$ winds found in $\mathrm{OH} / \mathrm{IR}$ stars. The superwind models for the dust shells around AGB stars may successfully explain those observations that require the density distributions that are not simple power laws (Suh \& Jones 1997; Suh 1997; and references therein).
During a pulsation cycle, the detailed dust density distributions near the dust formation radius are more complicated than simple power laws (Suh et al. 1990). However, at outer radii outside $3-5 R_{\mathrm{c}}$, the constant expansion velocity and continuous density distribution are maintained over a pulsation cycle. Using different density distributions near $R_{\mathrm{c}}$ have only minor effects on the emergent SEDs (Suh et al. 1990). Although small variations of inner radii produce very different output SEDs, detailed density variations near $R_{\mathrm{c}}$ do not significantly affect the emergent SEDs.

Both simple radiation pressure models (e.g. Kwok 1975; Kozasa et al. 1984) or models that add pulsation and shocks (Bowen 1988; Suh et al. 1990) predict overall $r^{-2}$ density laws. These models show that dust grains are accelerated relatively fast and approach and maintain terminal velocity within $3-5 R_{\mathrm{c}}$. Dust formation and growth time-scales are very short (about a week) compared with other time scales typical in OH/IR star winds (e.g. Suh et al. 1990). This burst-like grain formation implies a constant outflow velocity for most of the dust shell.

Considering above arguments, we expect that the overall density distribution of the dust shell at different phases follows roughly $r^{-2}$ density law even in complicated conditions due to pulsation. This expectation could be more acceptable when we consider the dust shell parameters that really affect the emergent spectra. Therefore, we assume $r^{-2}$ density law for deriving approximate global dust formation parameters at different phases.

\subsection{Evaporation of dust grains in the course from the minimum to maximum phase}

For both $\mathrm{OH} / \mathrm{IR}$ stars, it takes about 2.1 yrs from the minimum to maximum phase. The luminosity of the central star increases by more than a factor 3 and the inner radius of the dust shell should increase if the dust formation temperature is fixed. We expect that a significant amount of dust grains should be evaporated if the velocity of the moving inner shell radius due to pulsation $\left(v_{\mathrm{p}}\right)$ exceeds the constant expansion wind velocity $\left(v_{\exp }\right)$ that provides the materials for dust formation. If those speeds are the same, the dust formation ceases during the phase change from the minimum to maximum.

To estimate the amount of dust grains to be evaporated, we use simple approximations. We assume that the mass-loss rate $(\dot{M})$ and the envelope expansion velocity $\left(v_{\text {exp }}\right)$ are constant, and overall density distribution is continuous $\left(\rho \propto r^{-2}\right)$. 
Table 4. Dust shell parameters at the minimum and maximum phases: inner radii of the dust shell, dust mass in the volume difference, evaporated dust mass, and newly formed dust mass.

\begin{tabular}{ccccccccc}
\hline \hline $\begin{array}{c}\text { Object } \\
\text { Name }\end{array}$ & $\begin{array}{c}R_{0} \\
(\mathrm{~cm})\end{array}$ & $\begin{array}{c}R_{1} \\
(\mathrm{~cm})\end{array}$ & $\begin{array}{c}M_{\text {dust,vol }} \\
\left(M_{\odot}\right)\end{array}$ & $\begin{array}{c}T \\
(\mathrm{yrs})\end{array}$ & $\begin{array}{c}v_{\text {exp }} \\
\left(\mathrm{km} \mathrm{s}^{-1}\right)\end{array}$ & $\begin{array}{c}v_{\mathrm{p}} \\
\left(\mathrm{km} \mathrm{s}^{-1}\right)\end{array}$ & $\begin{array}{c}\Delta M_{\text {dust }} \\
\left(M_{\odot}\right) \\
\text { Min to Max }\end{array}$ & $\begin{array}{c}\Delta M_{\text {dust }} \\
\left(M_{\odot}\right) \\
\text { Max to Min }\end{array}$ \\
\hline OH127.8+0.0 & $3.34 \mathrm{E}+14$ & $5.96 \mathrm{E}+14$ & $2.70 \mathrm{E}-06$ & 2.104 & 12.5 & 39.5 & $-1.85 \mathrm{E}-06$ & $3.56 \mathrm{E}-6$ \\
OH26.5+0.6 & $3.46 \mathrm{E}+14$ & $5.69 \mathrm{E}+14$ & $3.07 \mathrm{E}-06$ & 2.134 & 14.0 & 33.0 & $-1.77 \mathrm{E}-06$ & $4.38 \mathrm{E}-6$ \\
\hline
\end{tabular}

Then the dust mass added from minimum phase to maximum phase is given by

$\Delta M_{\text {dust }}=4 \pi \int r^{2} \rho_{\mathrm{d}} v \mathrm{~d} t \simeq 4 \pi R_{0}^{2} \rho_{\text {od }} \int_{0}^{T}\left(v_{\text {exp }}-v_{\mathrm{p}}\right) \mathrm{d} t$,

where $\rho_{\text {od }}$ is the dust density at the inner radius of the dust shell at minimum phase $\left(R_{0}\right)$ and the velocity of the moving inner shell radius due to pulsation $\left(v_{\mathrm{p}}\right)$ is given by

$v_{\mathrm{p}}=\frac{R_{1}-R_{0}}{T}$,

where $T$ is the time required from the minimum to maximum phase and $R_{1}$ is the inner radius of the dust shell at maximum phase. We assume that $T$ is a half of the period of pulsation. If $v_{\mathrm{p}}$ is greater than $v_{\text {exp }}$, the mass of dust grains is decreasing and the value $\Delta M$ in Eq. (5) corresponds to the dust mass evaporated in $T$. Table 4 lists the parameters.

When the central luminosity increases from the minimum to maximum phase, we find that the inner radius of the dust shell increases with velocity faster than the speed of continuous expansion velocity and the shell optical depth decreases. During the phase change from the minimum to maximum, we find that dust formation ceases and about a half of the dust grains in the volume difference should be evaporated (see Table 4).

For both stars, about $2 \times 10^{-6} M_{\odot}$ of dust grains should be destroyed in about 2 yrs. Although our approximation of overall $r^{-2}$ density law may be too crude, this result will be meaningful in estimating the changes of the global dust shell parameters.

\subsection{Enhanced formation of dust grains in the course from the maximum to minimum phase}

In the course from the maximum to minimum phase, the luminosity of the central star decreases by more than a factor 3 and the inner radius of the dust shell should decrease if the dust formation temperature is fixed.

The dust mass that should be added from maximum phase to maximum phase is given by

$\Delta M_{\text {dust }} \simeq 4 \pi R_{0}^{2} \rho_{\text {od }} \int_{0}^{T}\left(v_{\text {exp }}+v_{\mathrm{p}}\right) \mathrm{d} t$.

This is the same formulation as Eq. (5) with opposite direction of $v_{\mathrm{p}}$. The last column of Table 4 lists the the dust mass. During the phase change from the maximum to minimum, the dust formation should be enhanced because the inner radius is
Table 5. Dust formation rates for a pulsation cycle.

\begin{tabular}{cccc}
\hline \hline $\begin{array}{c}\text { Object } \\
\text { Name }\end{array}$ & $\begin{array}{c}\dot{M}_{\text {dust }} \\
\text { Overall } \\
\left(M_{\odot} / \mathrm{yr}\right)\end{array}$ & $\begin{array}{c}\dot{M}_{\text {dust }} \\
\text { Min to Max } \\
\left(M_{\odot} / \mathrm{yr}\right)\end{array}$ & $\begin{array}{c}\dot{M}_{\text {dust }} \\
\text { Max to Min } \\
\left(M_{\odot} / \mathrm{yr}\right)\end{array}$ \\
\hline OH127.8+0.0 & $4.06 \mathrm{E}-07$ & $-8.79 \mathrm{E}-07$ & $+1.69 \mathrm{E}-06$ \\
OH26.5+0.6 & $6.11 \mathrm{E}-07$ & $-8.30 \mathrm{E}-07$ & $+2.05 \mathrm{E}-06$ \\
\hline
\end{tabular}

decreasing. In the outer radii of the dust shell, the constant dust winds are easily maintained.

Table 5 lists the dust formation rates at different phases. Over a pulsation cycle, the dust formation radius and the dust formation rates change. During the phase change from the minimum to maximum, dust formation ceases and a significant amount of dust grains should be evaporated. On the other hand, during the phase change from the maximum to minimum, there should be enhanced dust formation. However, outside the inner radius of the dust shell at the maximum phase, the dusty stellar winds supply gas and dust to the outer radii at constant rate (overall $\dot{M}_{\text {dust }}$ ). In outer regions of the dust shells around OH/IR stars, the constant dusty winds would be measured regardless of the pulsation phase.

\subsection{Crystallization of dust grains during a pulsation cycle}

Suh (2002) argued that a fraction of silicates initially condensed in an amorphous form may subsequently be annealed to become a crystalline form in the inner region of the outflowing envelope for the OH/IR stars with thick dust shells. The crystallization would be generally processed in the inner regions of outflowing spherical dust shells. If dust formation temperature is $1000 \mathrm{~K}$, the inner region of the outflowing envelope is hot (about 900-1000 K) during an extended period of time (several hundred days) for a known crystallization process - annealing.

In this paper we assume that dust formation temperature is $1000 \mathrm{~K}$ for all models. However, detailed investigations about dust formation processes suggested that the dust formation temperature is higher for more dusty stars with stronger stellar winds (e.g. Kozasa et al. 1984; Gail \& Sedlmayr 1999; Sogawa \& Kozasa 1999). If the dust formation temperature is much lower than $1000 \mathrm{~K}$ for low mass-loss rate O-rich AGB stars, the annealing would not be effective for those stars. This may be the reason why we find virtually no evidence of crystalline 
silicates for low mass-loss rate AGB stars. If the dust formation temperature is higher than $1000 \mathrm{~K}$ for higher mass-loss rate Orich AGB stars, the annealing process would be more effective in crystallizing amorphous silicates.

Based on the arguments presented in this paper, we expect that the dust evaporation processes driven by pulsation could be a mechanism for crystallizing dust grains in inner shells of $\mathrm{OH} / \mathrm{IR}$ stars. One may think that a significant amount of dust grains, which was originally in an amorphous form, was partially evaporated in the course from the minimum to maximum phase. Those partially evaporated dust grains will be in a crystalline form. Therefore, the OH/IR stars with higher amplitude pulsations can produce more crystalline dust grains in their dust shells. Crystalline grains could be produced more efficiently in the course of the phase change from minimum to maximum because of the evaporation occurs only in that interval. In outer regions of a dust shell, crystalline grains could be well mixed with amorphous grains.

For OH/IR stars with thick dust shells, we expect that the variations of the crystallization process depending on the pulsation phase would not be detectable through the emergent spectra. This could be verified when we get more observational data as good as those from ISO at different phases and a radiative transfer model that can deal with detailed radial variations of dust chemistry.

\section{Conclusions}

In this paper, we have investigated SEDs of OH/IR stars (OH 127.8+0.0 and $\mathrm{OH} 26.5+0.6)$ with thick dust envelopes at different pulsation phases. We have drawn the following conclusions.

1. Using new infrared observational data including ISO, we have determined the new pulsation parameters (period and amplitude).

2. We have found that when the central luminosity increases from the minimum to maximum phase, the dust shell inner radius increases with a velocity faster than the outer shell expansion velocity and the dust shell optical depth decreases.

3. During the phase change from the minimum to maximum phase, we have found that dust formation ceases and about a half of the dust grains in the volume difference should be evaporated.

4. During the phase change from the maximum to minimum phase, we have found that the dust formation should be enhanced because the inner radius is decreasing. In the outer radii of the dust shell, the constant dust winds are easily maintained.

5. We expect that the dust evaporation process driven by pulsation could be a mechanism for crystallizing the dust grains in inner regions of the dust shells around OH/IR stars.

Acknowledgements. We thank the anonymous referee for constructive comments. The authors are grateful to F. Kemper who provided the reduced $I S O$ spectroscopic data for the two OH/IR stars. This work was supported by Korea Research Foundation Grant (KRF-2000-015DP0445).

\section{References}

Bedijn, P. J. 1987, A\&A, 186, 136

Bowen, G. H. 1988, ApJ, 329, 299

Dyck, H. M., Zuckerman, B., Leinert, Ch., \& Beckwith, S. 1984, ApJ, 287,801

Egan, M. P., Leung, C. M., \& Spagna, G. F. Jr. 1988, Comp. Phys. Comm., 48, 271

Engels, D. 1982, Veröff. Astron. Inst. Bonn, 95

Evans II, N. J., \& Beckwith, S. 1977, ApJ, 217, 729

Forrest, W. J., Houck, J. R., McCarthy, J. F., et al. 1978, ApJ, 219, 114

Gail, H.-P., \& Sedlmayr, E. 1999, A\&A, 347, 594

Gehrz, R. D., Hackwell, J. A., Grasdalen, G. L., Kleinmann, S. G., \& Mason, S. 1985, ApJ, 290, 296

Gezari, D. Y., Pitts, P. S., \& Schmitz, M. 2000, Catalog of Infrared Observation, Version 5.1, NASA Reference

Grasdalen, G. L., Gehrz, R. D., Hackwell, J. A., et al. 1983, ApJS, 53, 413

Groenewegen, M. A. T., Baas, F., Blommaert, J. A. D. L., et al. 1999, A\&AS, 140, 197

Herman, J., \& Habing, H. J. 1985, A\&AS, 59, 523

Jäger, C., Molster, F. J., Dorschner, J., et al. 1998, A\&A, 339, 904

Joint IRAS Science Working Group, 1986a, IRAS catalogs and atlases, Point Source Catalog (PSC), US Government Printing Office, Washington

Joint IRAS Science Working Group, 1986b, IRAS catalogs and atlases, Low Resolution Spectrograph (LRS), A\&AS, 65, 607

Jones, T. J., Bryja, C. O., Gehrz, R. D., et al. 1990, ApJS, 74, 785

Jones, T. W., \& Merrill, K. M. 1976, ApJ, 209, 509

Kozasa, T., Hasegawa, H., \& Seki, J. 1984, Ap\&SS, 98, 61

Kwok, S. 1975, ApJ, 198, 583

Le Bertre, T. 1988, A\&A, 190, 79

Lebofsky, M. J., Sargent, D. G., Kleinmann, S. G., \& Rieke, G. H. 1978, ApJ, 219, 487

Lepine, J. R. D., Ortiz, R., \& Epchtein, N. 1995, A\&A, 299, 453

Le Sidaner, P., \& Le Bertre, T. 1993, A\&A, 278, 167

Le Sidaner, P., \& Le Bertre, T. 1996, A\&A, 314, 896

Lobel, A., Bagnulo, S., Doyle, J. G., \& Power, C. 2000, MNRAS, 317, 391

Loup, C., Forveille, T., Omont, A., \& Paul, J. F. 1993, A\&AS, 99, 291

Monnier, J. D., Geballe, T. R., \& Danchi, W. C. 1998, ApJ, 502, 833

Ney, E. P., \& Merrill, K. M. 1980, Study of Sources in AFGL Rocket Infrared Study, Air Force Geophysical Laboratory, AFGL-TR-800050

Nyman, L.-A., Hall, P. J., \& Le Bertre, T. 1993, A\&A, 280, 551

Olivier, E., Whitelock, P., \& Marang, F. 2001, MNRAS, 326, 490

Persi, P., Ferrari-Toniolo, M., Ranieri, M., Marenzi, A., \& Shivanandan, K. 1990, A\&A, 237, 153

Press, W. H., Flannery, B. P., Teukolsy, S. A., \& Vetterling, W. T. 1986, Numerical Recipes (Cambridge: Cambridge Unversity Press)

Rowan-Robinson, M., \& Harris, S. 1983, MNRAS, 202, 767

Sogawa, H., \& Kozasa, T. 1999, ApJ, 516, L33

Suh, K.-W., Jones, T. J., \& Bowen, G. H. 1990, ApJ, 358, 588

Suh, K.-W., \& Jones, T. J. 1997, ApJ, 479, 918

Suh, K.-W. 1997, MNRAS, 289, 559

Suh, K.-W. 1999, MNRAS, 304, 389

Suh, K.-W. 2002, MNRAS, 332, 513

Sylvester, R. J., Skinner, C. J., \& Barlow, M. J. 1998, MNRAS, 301, 1083

Sylvester, R. J., Kemper, F., Barlow, M. J., et al. 1999, A\&A, 352, 587

Volk, K., \& Kwok, S. 1988, ApJ, 331, 435

Werner, M. W., Beckwith, S., Gatley, I., et al. 1980, ApJ, 239, 540

Willems, F., \& De Jong, T. 1982, A\&A, 115, 213

Xiong, G. Z., Chen, P. S., \& Gao, H. 1994, A\&AS, 108, 661 